\title{
Brain structure and cognition 3 years after the end of an early menopausal hormone therapy trial
}

Kejal Kantarci, MD, MS, Nirubol Tosakulwong, Timothy G. Lesnick, MS, Samantha M. Zuk, Val J. Lowe, MD, Julie A. Fields, PhD, Jeffrey L. Gunter, PhD, Matthew L. Senjem, MS, Megan L. Settell, Carey E. Gleason, PhD, Lynne T. Shuster, MD, Kent R. Bailey, PhD, N. Maritza Dowling, PhD, Sanjay Asthana, MD, Clifford R. Jack, Jr., MD, Walter A. Rocca, MD, MPH, and Virginia M. Miller, PhD

Neurology ${ }^{\circledR}$ 2018;90:e1404-e1412. doi:10.1212/WNL.0000000000005325

\begin{abstract}
Objective

The effects of 2 frequently used formulations of menopausal hormone therapy ( $\mathrm{mHT}$ ) on brain structure and cognition were investigated 3 years after the end of a randomized, placebocontrolled trial in recently menopausal women with good cardiovascular health.

\section{Methods}

Participants (aged 42-56 years; 5-36 months past menopause) were randomized to one of the following: $0.45 \mathrm{mg} / \mathrm{d}$ oral conjugated equine estrogen (oCEE); $50 \mu \mathrm{g} / \mathrm{d}$ transdermal $17 \beta$-estradiol (tE2); or placebo pills and patch for 4 years. Oral progesterone $(200 \mathrm{mg} / \mathrm{d})$ was given to mHT groups for 12 days each month. MRIs were performed at baseline, at the end of 4 years of $\mathrm{mHT}$, and 3 years after the end of $\mathrm{mHT}(\mathrm{n}=75)$. A subset of participants also underwent Pittsburgh compound B-PET $(n=68)$.
\end{abstract}

\section{Results}

Ventricular volumes increased more in the oCEE group compared to placebo during the 4 years of $\mathrm{mHT}$, but the increase in ventricular volumes was not different from placebo 3 years after the discontinuation of mHT. Increase in white matter hyperintensity volume was similar in the oCEE and tE2 groups, but it was statistically significantly greater than placebo only in the oCEE group. The longitudinal decline in dorsolateral prefrontal cortex volumes was less in the tE2 group compared to placebo, which correlated with lower cortical Pittsburgh compound B uptake. Rates of global cognitive change in mHT groups were not different from placebo.

\section{Conclusions}

The effects of oCEE on global brain structure during mHT subside after oCEE discontinuation but white matter hyperintensities continue to increase. The relative preservation of dorsolateral prefrontal cortical volume in the $\mathrm{tE} 2$ group over 7 years indicates that $\mathrm{mHT}$ may have long-term effects on the brain.

\section{Classification of evidence}

This study provides Class III evidence that the rates of change in global brain volumes and cognitive function in recently menopausal women receiving $\mathrm{mHT}$ ( $\mathrm{tE} 2$ or oCEE) were not significantly different from women receiving placebo, as measured 3 years after exposure to mHT.

\author{
Correspondence \\ Dr. Kantarci \\ kantarci.kejal@mayo.edu
}

\section{MORE ONLINE}

$\rightarrow$ Class of Evidence

Criteria for rating

therapeutic and diagnostic studies

NPub.org/coe 


\section{Glossary}

KEEPS $=$ Kronos Early Estrogen Prevention Study; $\mathrm{mHT}=$ menopausal hormone therapy; $\mathrm{oCEE}=$ oral conjugated equine estrogen; PiB = Pittsburgh compound B; ROI = region of interest; SUVR = standard unit value ratio; tE2 = transdermal $17 \beta$ estradiol; WHIMS = Women's Health Initiative Memory Study; WMH = white matter hyperintensity.

Based on findings from observational studies, the timing hypothesis posits that estrogen may preserve neurologic function and decrease the risk of dementia when administered early in menopause; however, the effects may be neutral or even harmful if estrogen is initiated later in life. $^{1-14}$ Determining the effects of early menopausal hormone therapy $(\mathrm{mHT})$ on the risk of dementia in later life would require decades of follow-up; alternatively, noninvasive imaging biomarkers can potentially assess the efficacy of preventive interventions for cognitive decline and dementia in younger cohorts.

Oral conjugated equine estrogen (oCEE) and transdermal $17 \beta$-estradiol (tE2) therapy administered within 3 to 36 months after the onset of menopause in the Kronos Early Estrogen Prevention Study (KEEPS) ${ }^{15}$ did not have an effect on biomarkers of atherosclerosis or cognitive function during the 4 years of $\mathrm{mHT} .{ }^{16,17}$ On the contrary, the ancillary MRI study of the brain in a subset of the KEEPS participants found greater expansion of the ventricles in the oCEE group compared to placebo, and trends for white matter hyperintensities (WMH) to increase in both the oCEE and tE2 groups during the 4 years of $\mathrm{mHT}^{18}{ }^{18}$ Women who participated in the ancillary KEEPS MRI study were evaluated again 3 years after the end of $\mathrm{mHT}$ for global cognitive function and structural brain MRI. A subset of the participants also underwent PET imaging using the Pittsburgh compound B (PiB) ligand for $\beta$-amyloid, which showed lower PiB uptake in the tE2 group compared to placebo in an earlier analysis. ${ }^{19}$ Herein, we report longitudinal changes in structural brain MRI and global cognitive function 3 years after the end of mHT in KEEPS, and the association of these findings with $\beta$-amyloid load on PET.

\section{Methods}

\section{Participants}

KEEPS was a multicenter, randomized, double-blinded, placebo-controlled trial of 2 frequently used formulations of mHT in recently menopausal women with good cardiovascular health $(\mathrm{n}=727)$. Participants enrolled in KEEPS were between 42 and 59 years of age and they were within 5 to 36 months past their last menses. ${ }^{15}$ An ancillary MRI study to KEEPS was conducted during the 4 years of $\mathrm{mHT}$ and also 3 years after the end of $\mathrm{mHT}$ at the Mayo Clinic enrollment site to investigate the effects of $\mathrm{mHT}$ on brain structure and cognitive function. ${ }^{18} \mathrm{~A}$ subset of participants also underwent $\beta$-amyloid PiB-PET imaging. ${ }^{19}$ Participants were excluded from the ancillary KEEPS-MRI study if they had contraindications for MRI safety or neurologic disorders.
MRI examinations and cognitive testing were completed before randomization (baseline) and following randomization to mHT: (1) oCEE (Premarin, $0.45 \mathrm{mg} / \mathrm{d}$ ); (2) tE2 (skin patch, Climara, $50 \mu \mathrm{g} / \mathrm{d}$ ); or (3) placebo pills and patch. To protect the endometrium, progesterone was given orally (Prometrium; micronized progesterone, $200 \mathrm{mg} / \mathrm{d}$ ) for 12 days at the beginning of each month to both mHT groups, and placebo pill was given to the placebo group. MRI examinations and cognitive testing were performed at 18,36 , and 48 months during the mHT. ${ }^{15}$ For the current study, MRI and the cognitive tests were repeated at 3 years following the end of the mHT phase, which corresponded to 84 months (7 years) after randomization. All participants were genotyped for the APOE $\varepsilon 4$ allele.

\section{Standard protocol approvals, registrations, and patient consents}

The present study (NCT00154180; clinicaltrials.gov/ct2/ show/NCT00154180) was approved by the Mayo Clinic institutional review board (no. 224104). All participants provided written informed consent.

\section{Cognitive testing}

To assess the underlying structure of baseline cognitive data from the KEEPS Cognitive and Affective Study (NCT00623311), a confirmatory factor analysis was used and summary scores were derived $(n=662){ }^{20}$ The cognitive variables were summarized in a general domain representing global cognitive function using standard criteria for model fit. ${ }^{17}$

\section{Magnetic resonance imaging}

All MRI studies were performed on a single 1.5-tesla system, with an 8-channel phased-array coil (GE Healthcare, Waukesha, WI). A 3-dimensional (3D) T1-weighted sequence was acquired for volumetric analysis and fluid attenuated inversion recovery-MRI was acquired for quantification of WMH volume. Changes in ventricle and whole brain volumes were calculated automatically from each registered 3D T1-weighted scan pair using the boundary shift integral and were expressed in cubic centimeters of volume change from baseline for each follow-up time point. ${ }^{21} \mathrm{WMH}$ volumes were derived from semiautomated segmentation of fluid attenuated inversion recovery images as previously described. ${ }^{22}$ Annual percent change in volume was calculated for anatomical structures such as the whole brain and ventricular volumes. Total change in volume was calculated for the WMH lesion load.

Regional change in cortical volumes by 84 months was estimated with an automated, in-house-developed implementation of tensor-based morphometry using symmetric 
diffeomorphic normalization. ${ }^{23}$ This method uses the symmetric diffeomorphic registration between serial T1-weighted MRIs. The annualized log determinants of the Jacobians of these deformations were determined for each voxel. An inhouse-modified atlas of 21 regions of interest (ROIs) that covered the entire cortex in both hemispheres ${ }^{24}$ was applied to the annualized log Jacobian images to determine the magnitude of cortical volume change from baseline in each ROI.

\section{PiB-PET}

A PET/CT scanner (DRX; GE Healthcare) operating in 3D mode was used. Participants $(n=68)$ were injected with an average of $596 \mathrm{MBq}{ }^{11} \mathrm{C}-\mathrm{PiB}$ (range, 292-729 MBq). After a 40-minute uptake period, a 20-minute scan consisting of four 5-minute dynamic frames was obtained. PiB-PET quantitative analysis was performed using the fully automated image processing pipeline as previously described, ${ }^{25}$ and $\mathrm{PiB}$ retention was calculated by the $\mathrm{PiB}$ standard unit value ratio (SUVR) referenced to cerebellar gray matter.

\section{Statistical analysis}

Baseline characteristics were compared among oCEE, tE2, and placebo groups using analysis of variance followed by Tukey pairwise tests, or Fisher exact tests, as appropriate. In the descriptive table, the pairwise test results are reported for each of the treatment groups compared to placebo when significant. Changes in the outcomes from baseline were shown using plots of mean values and $95 \%$ confidence intervals at each time point. Associations across the outcome variables were assessed using scatterplots with Pearson correlation coefficients and their associated $p$ values.

The annual percent change in ventricular and whole brain volumes, WMH volumes, and global cognitive scores over time were modeled to compare each of the $\mathrm{mHT}$ treatment groups to placebo using linear mixed models and by including random subject-specific intercepts, slopes, and treatment $x$ time interactions. The classification of evidence was Class III for the primary research objective. To investigate the changes that occurred before and after mHT, each of the oCEE and tE2 groups was compared to placebo on the annual percent change in whole brain and ventricular volumes, total change in WMH volumes, and global cognition scores by $t$ tests. There was no adjustment for multiple comparisons in reporting these results, because the analyses on the effects of each of the 2 mHT formulations on brain structure was determined a priori. Furthermore, results from the $2 \mathrm{mHT}$ groups were compared to each other, but not pooled because of the differences in formulations and route of administration.

Regional cortical volume changes in each of the oCEE and tE2 groups were compared to placebo using area under the receiver operating characteristic curve analysis for 21 ROIs combining the right and left hemispheric regions. Because 21 ROIs were compared to placebo, the analyses were corrected for multiple comparisons by using the false discovery rate.

\section{Results}

All women enrolled in KEEPS at the Mayo Clinic in Rochester, MN ( $\mathrm{n}=118)$, were invited to participate in the ancillary KEEPS-MRI study. Twelve women declined participation in MRI and 5 participants were excluded because of neurologic disorders or MRI contraindications. Of the participants who underwent an MRI at baseline $(n=101)$, those who completed a follow-up MRI 3 years after the end of the mHT phase $(n=75 ; 84$ months after the baseline evaluation; figure e-1, links.lww.com/WNL/A366) were included. At baseline, cardiovascular risk factors and global cognitive performance were not different among women assigned to either of the $2 \mathrm{mHT}$ groups compared to placebo. However, women assigned to the tE2 group had a higher frequency of APOE \&4 carriers (48\%) compared to those assigned to placebo $(16 \% ; p=0.03)$. None of the participants had silent infarcts on baseline and follow-up MRI examinations. However, the WMH volume at baseline was higher in the tE2 group compared to placebo after adjusting for the logtransformed total intracranial volume $(p=0.04)$ (table 1$)$. These characteristics were not different between women who completed the follow-up at 3 years after the end of the $\mathrm{mHT}$ phase and those who did not complete the follow-up and, therefore, were excluded from the current study (table e-1, links.lww.com/WNL/A367).

The results of linear mixed-effects models are summarized in table 2. Treatment $x$ time interactions indicated that the WMH volume increased faster in the oCEE group compared to placebo $(p<0.05)$. Figure 1 shows the trajectories of structural brain changes and changes in global cognitive function in the $\mathrm{tE} 2$, oCEE, and placebo groups. The whole brain volumes showed a decline in the oCEE group more than the placebo group during the 48 months of the $\mathrm{mHT}$ phase. However, this trend was reversed, and the decline in whole brain volumes was less than in the placebo after the mHT ended $(p=0.02)$. Overall, the decline in whole brain volumes was not different between the oCEE and placebo groups by 84 months. Similarly, increases in ventricular volume during the 48 months of the $\mathrm{mHT}$ phase were greater in the oCEE group compared to the placebo, but this increase slowed after the end of mHT, and was not different from placebo by 84 months after starting mHT. The mean increases in $\mathrm{WMH}$ volume from baseline were greater in the oCEE group compared to placebo by 84 months $(p=0.03)$. The increases in WMH did not reach statistical significance in the tE2 group compared to placebo $(p=0.17)$ (table e-2, links.lww.com/WNL/A367). There were no differences in global cognitive $z$ score change in the oCEE and tE2 groups compared to placebo by 84 months. Furthermore, no correlation was observed between the change in global cognitive $z$ score and the change in ventricular $(p=0.94)$, whole brain $(p=0.77)$, and WMH $(p=0.92)$ volumes after adjusting for age. The changes in global cognitive scores, ventricular, whole brain, and WMH volumes did not differ between the oCEE and tE2 groups. 
Table 1 Baseline characteristics of the participants

\begin{tabular}{|c|c|c|c|}
\hline Characteristic & OCEE $(n=20)$ & tE2 $(n=22)$ & Placebo $(n=33)$ \\
\hline Age at baseline, $y$ & $53(2)$ & $53(3)$ & $53(2)$ \\
\hline \multicolumn{4}{|l|}{ Education, n (\%) } \\
\hline High school or less & $1(6)$ & $1(5)$ & $3(9)$ \\
\hline Some college/college graduate & $14(78)$ & $13(65)$ & $19(58)$ \\
\hline Some graduate/graduate & $3(17)$ & $6(30)$ & $11(33)$ \\
\hline \multicolumn{4}{|l|}{ Smoking status, n (\%) } \\
\hline Nonsmoker & $15(75)$ & $13(59)$ & $24(73)$ \\
\hline Smoker (past or current) & $5(25)$ & $9(41)$ & $9(27)$ \\
\hline APOE $\varepsilon 4$ carrier, $\mathrm{n}(\%)$ & $3(15)$ & $10(48)^{a}$ & $5(16)$ \\
\hline Global cognitive function $z$ scores & $-0.20(0.77)$ & $0.07(0.79)$ & $0.15(0.67)$ \\
\hline Mean systolic blood pressure, $\mathrm{mm} \mathrm{Hg}$ & $122(13)$ & $116(14)$ & $122(12)$ \\
\hline Mean diastolic blood pressure, $\mathrm{mm} \mathrm{Hg}$ & $77(7)$ & $72(7)$ & $75(7)$ \\
\hline Waist circumference, $\mathrm{cm}$ & $80(22)$ & $82(13)$ & $84(11)$ \\
\hline Body mass index, $\mathrm{kg} / \mathrm{m}^{2}$ & $27.8(4.6)$ & $25.9(4.6)$ & $27.1(3.6)$ \\
\hline Low-density lipoprotein, mg/dL & $121(27)$ & $121(34)$ & $115(29)$ \\
\hline High-density lipoprotein, mg/dL & $69(10)$ & $71(11)$ & $70(14)$ \\
\hline Triglycerides, mg/dL & $80(49)$ & $90(44)$ & $83(46)$ \\
\hline Whole brain, $\mathrm{cm}^{3}$ & $1,349(68)$ & $1,322(95)$ & $1,288(83)$ \\
\hline White matter hyperintensities, $\mathrm{cm}^{3}$ & $2.5(1.3)$ & $2.6(1.7)^{a}$ & $1.5(0.7)$ \\
\hline
\end{tabular}

Abbreviations: OCEE: oral conjugated equine estrogen; tE2: transdermal 17 $\beta$-estradiol.

Data are $\mathrm{n}(\%)$ or mean (SD).

a Pairwise comparison to placebo $p<0.05$; pairwise $p$ values were assessed using Fisher exact and analysis of variance followed by Tukey pairwise tests.

Although the tE2 group had greater WMH volumes at baseline compared to placebo, accounting for the WMH volumes at baseline did not alter the differences between the
mHT groups (oCEE and tE2) compared to placebo (supplemental results, links.lww.com/WNL/A368). A subset of participants $(\mathrm{n}=11)$ continued to receive $\mathrm{mHT}$ for a range of

Table 2 Change in imaging markers and global cognition in the linear mixed-effects models, comparing oCEE and tE2 groups to placebo

\begin{tabular}{lllll}
\hline & Estimates $(95 \% \mathrm{Cl})$ & & & \\
\cline { 2 - 5 } & Whole brain, $\mathbf{c m}^{\mathbf{3}}$ & Ventricle, $\mathbf{c m}^{\mathbf{3}}$ & WMH, $\mathbf{c m}^{\mathbf{3}}$ & Global cognition \\
\hline Intercept & $1,289(1,260,1,318)^{\mathrm{a}}$ & $15.2(12.4,17.9)^{\mathrm{a}}$ & $1.51(1.28,1.75)^{\mathrm{a}}$ & $0.16(-0.98,1.31)$ \\
\hline Time from baseline & $-2.07(-2.96,-1.19)^{\mathrm{a}}$ & $0.35(0.21,0.50)^{\mathrm{a}}$ & $0.07(0.04,0.10)^{\mathrm{a}}$ & $0.04(-0.12,0.19)$ \\
\hline oCEE & $59.4(17.6,101.1)^{\mathrm{b}}$ & $3.36(-1.15,7.87)$ & $0.98(0.36,1.59)^{\mathrm{b}}$ & $-0.42(-2.33,1.48)$ \\
\hline tE2 & $34.0(-15.4,83.4)$ & $5.92(1.53,10.3)^{\mathrm{b}}$ & $1.03(0.29,1.77)^{\mathrm{b}}$ & $-0.08(-1.91,1.75)$ \\
\hline oCEE $\times$ time from baseline & $-0.20(-1.45,1.05)$ & $0.13(-0.11,0.36)$ & $0.08(0.01,0.15)^{\mathrm{c}}$ & $-0.07(-0.34,0.19)$ \\
\hline tE2 $\times$ time from baseline & $-0.64(-1.94,0.67)$ & $0.13(-0.10,0.36)$ & $0.07(-0.03,0.18)$ & $-0.06(-0.31,0.19)$
\end{tabular}

Abbreviations: $\mathrm{Cl}$ = confidence interval; oCEE = oral conjugated equine estrogen; tE2 = transdermal 17ß-estradiol; WMH = white matter hyperintensity Coefficient estimates with their associated $95 \% \mathrm{Cls}$ and categories of significance are reported for these models. Treatment $\times$ time interactions were of interest because any treatment effects would likely manifest as a change in the trajectories over time.

a $p<0.001$.

b $p<0.01$

$c p<0.05$. 
A

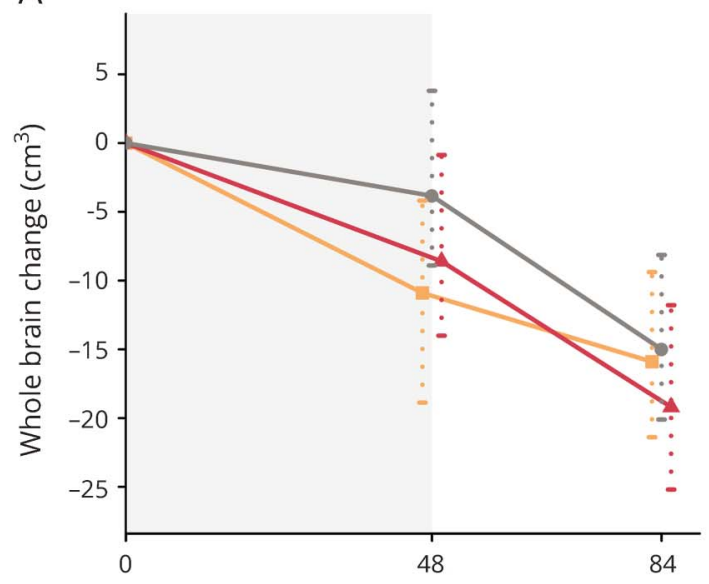

C

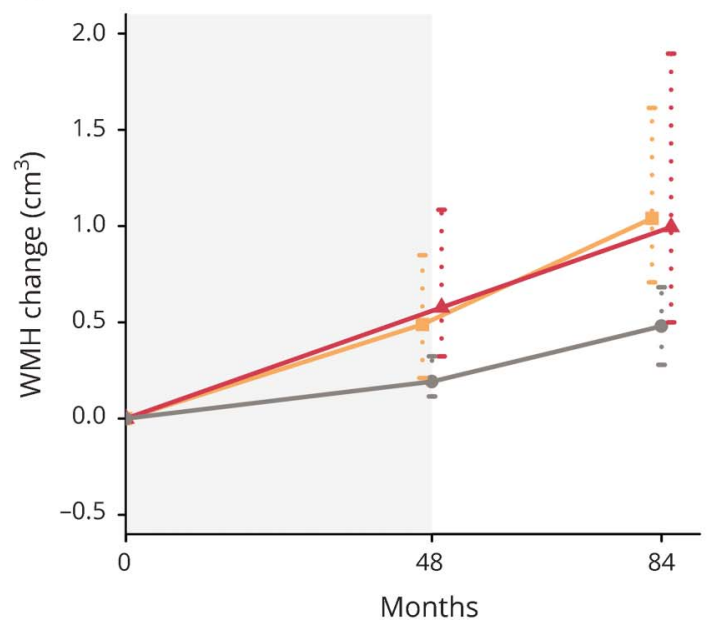

B

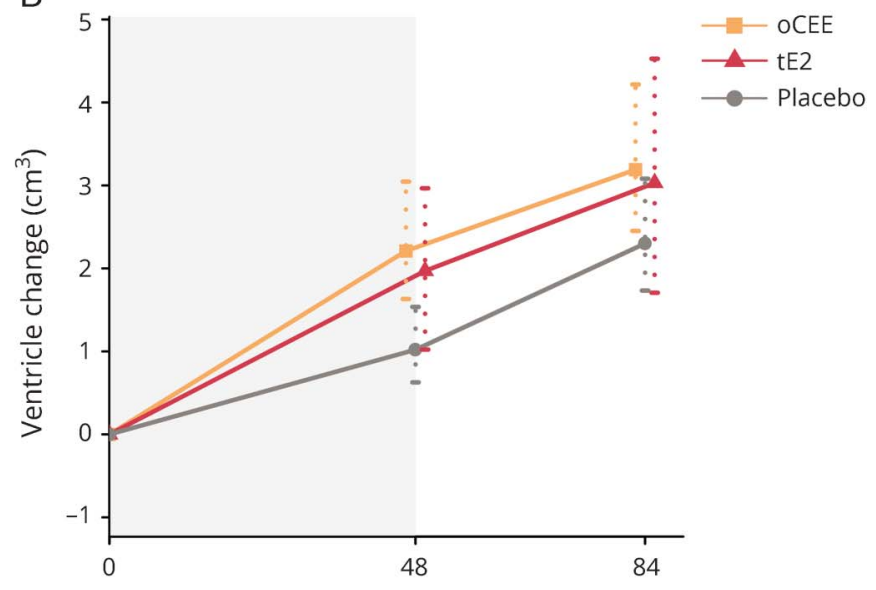

D

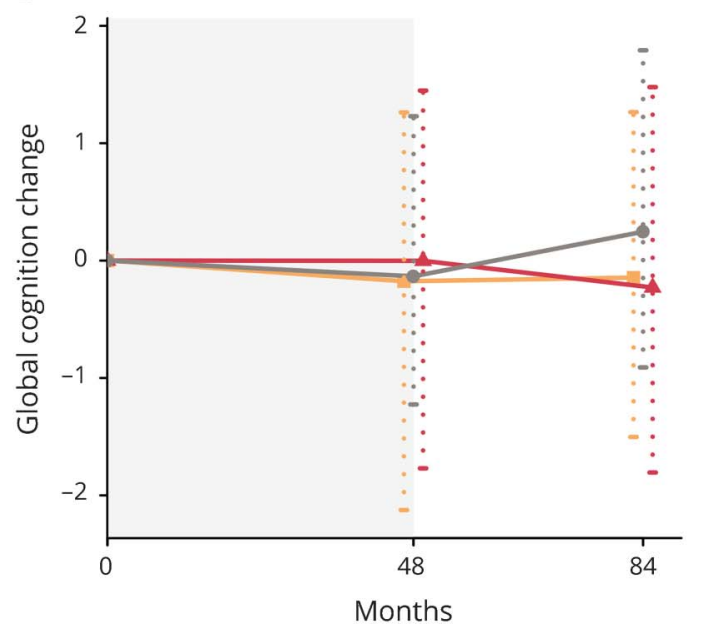

Longitudinal change in global brain structure and global cognitive function ([A] whole brain, $[\mathrm{B}]$ ventricle, $[\mathrm{C}] \mathrm{WMH},[\mathrm{D}]$ global cognition) in the tE2, oCEE, and placebo groups during $\mathrm{mHT}$ (0-48 months) and during the 3 years after mHT (48-84 months). Shaded panels represent the clinical trial phase. $\mathrm{mHT}=$ menopausal hormone therapy; oCEE = oral conjugated equine estrogen; tE2 = transdermal $17 \beta$-estradiol; $\mathrm{WMH}=$ white matter hyperintensity.

1 to 13 months after the end of the mHT phase of the trial. Two of the participants (one in the oCEE group and one in the placebo group) were taking oCEE, and 9 of the participants were taking tE2 ( 4 in the oCEE group and 5 in the tE2 group) during this time frame. A sensitivity analysis after removing these individuals did not alter the findings (data not shown).

A total of 21 ROIs, which covered the entire cerebral cortex (averaged for the right and left hemispheres), were analyzed to determine the differences in the rate of regional cortical volume change over the 84 months of follow-up. Lower annualized rates of decrease in superior and middle frontal gyri cortical volume were observed in the tE2 group compared to placebo ( $p=0.04$; false discovery rate-corrected for multiple comparisons) (figure e-2, links.lww.com/WNL/ A366). No differences in the rates of regional cortical volume change were observed between oCEE and placebo groups (figure 2). Superior and middle frontal gyri cortical volumes were summed to investigate the association of the rates of cortical volume change in the composite ROI with $\mathrm{PiB}$ SUVR at 84 months and with the change in global cognitive function by 84 months. Greater preservation of the superior and middle frontal gyri cortical volumes correlated with lower PiB SUVR only in the tE2 group $(r=-0.51$; $p=0.02)$, but not in the oCEE $(r=0.16 ; p=0.54)$ or placebo ( $r=0.02 ; p=0.93$ ) group, and the APOE $\varepsilon 4$ status did not modify these correlations $(p>0.85$; figure 3$)$. The correlation between the superior and middle frontal gyri cortical preservation with lower PiB SUVR in the tE2 group attenuated after adjusting for age $(r=-0.42 ; p=0.07)$. Furthermore, no correlation was found between the change in superior and middle frontal gyri cortical volume and global cognitive function in the tE2 $(r=0.25 ; p=0.27)$, oCEE $(r=0.31 ; p=0.22)$, or placebo $(r=0.03 ; p=0.86)$ group. 

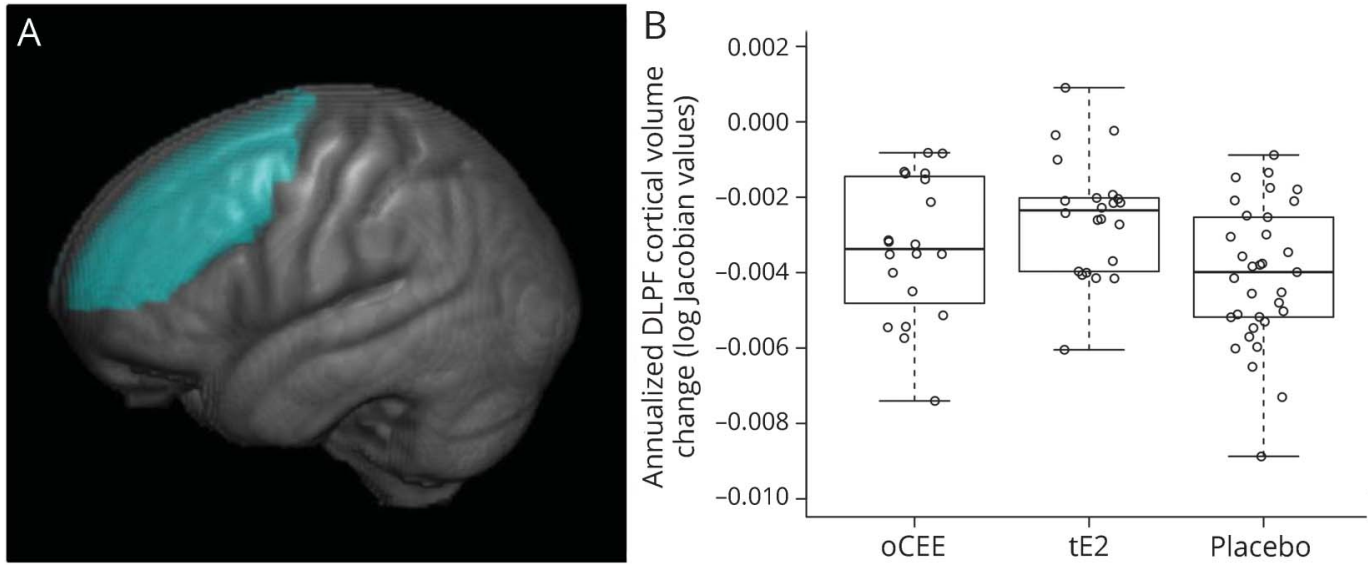

(A) Location of the superior and middle frontal gyri regions of interest (i.e., dorsolateral prefrontal cortex) is shown on a 3-dimensional surface render of the template brain. (B) Log Jacobian values for the annualized change in superior and middle frontal gyri cortical volumes are displayed in box-and-whisker plots. $\mathrm{DLPF}=$ dorsolateral prefrontal; oCEE = oral conjugated equine estrogen; tE2 = transdermal 17 $\beta$-estradiol.

\section{Discussion}

The main finding of this study is that the rates of change in global brain volumes and cognitive function in women randomized to 2 commonly used formulations of $\mathrm{mHT}$ ( $\mathrm{tE} 2$ or oCEE) did not differ from placebo 3 years after exposure to $\mathrm{mHT}$. WMH volume increased in both the oCEE and tE2 groups, but the rates of increase in WMH volume was statistically significantly greater than placebo in the oCEE group but not in the tE2 group. Dorsolateral prefrontal cortex volumes were preserved in the tE2 group relative to placebo during the treatment and in the 3 years following treatment. Furthermore, greater preservation of the dorsolateral prefrontal cortical volume correlated with lower global cortical $\beta$-amyloid deposition in the $\mathrm{tE} 2$ group.

During the 4 years of the $\mathrm{mHT}$ in KEEPS, the global changes in brain structure measured with ventricular volumes showed greater rates of expansion in the oCEE group compared to the placebo group. ${ }^{18}$ However, 3 years after the end of mHT, the rate of ventricular expansion was no longer different from the placebo group, suggesting that a higher rate of change in

Figure 3 Correlations between PiB SUVR (i.e., $\beta$-amyloid) deposition and reduction of cortical volume in superior and middle frontal gyri

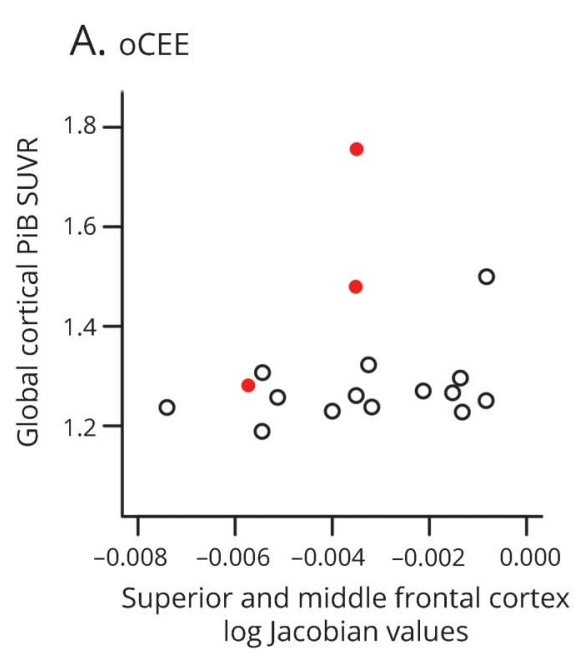

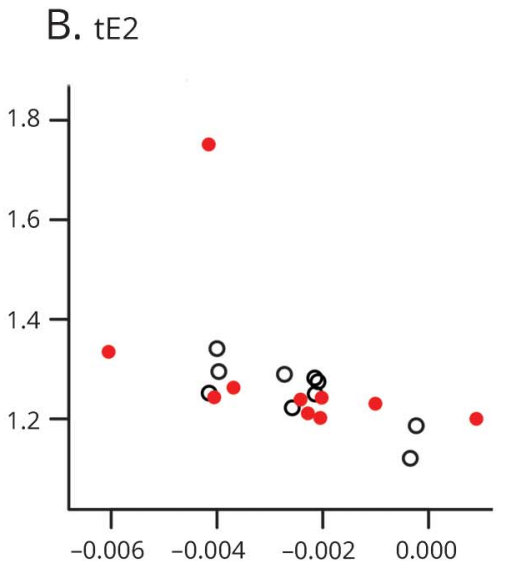

Superior and middle frontal cortex log Jacobian values

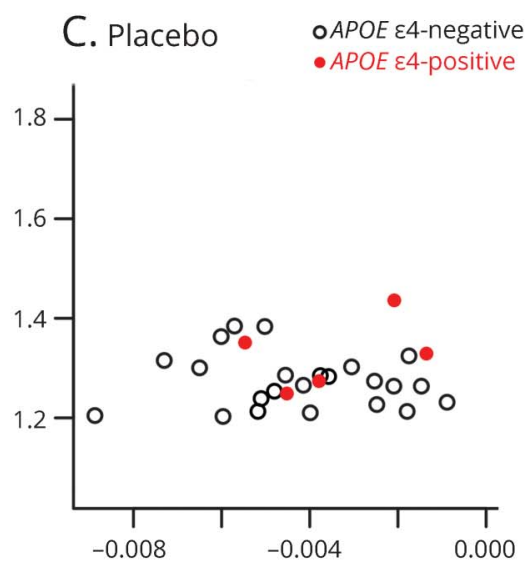

Superior and middle frontal cortex log Jacobian values

Correlation of global cortical PiB SUVR with the log Jacobian values of the annualized change in superior and middle frontal gyri cortical volumes 7 years after randomization in the (A) oCEE $(n=17),(B)$ tE2 $(n=21)$, and $(C)$ placebo $(n=30)$ groups. Participants who were APOE \&4 positive are plotted with red circles. A correlation was observed in the tE2 $(r=-0.51 ; p=0.02)$, but not in the OCEE and placebo groups. Removal of the outlier in the tE2 group strengthened the correlation but did not change the findings $(r=-0.74 ; p \leq 0.001)$. The correlations were not modified by $A P O E$ \& . oCEE $=$ oral conjugated equine estrogen; PiB = Pittsburgh compound B; SUVR = standard unit value ratio; tE2 = transdermal 17 $\beta$-estradiol. 
brain structure in the oCEE group was a transient effect that occurred only during the mHT phase as shown previously, ${ }^{18}$ and did not persist after $\mathrm{mHT}$ was discontinued. The finding of normalization of changes in brain structure to those observed in the placebo group after cessation of the mHT confirms that the accelerated brain structural changes observed in the oCEE group during the $\mathrm{mHT}$ phase were directly caused by oCEE. Furthermore, these findings are consistent with the Women's Health Initiative Memory Study (WHIMS)-MRI, in which the effects of mHT on brain volumes of older women were present soon after the oCEE treatments, ${ }^{26}$ but did not continue to decline faster than placebo after the end of oCEE therapies. ${ }^{27}$ The biological basis of structural brain changes during oCEE treatments needs further investigation.

The rates of global cognitive function change did not differ from placebo by 7 years, which is consistent with the changes observed during the mHT phase in the KEEPS-cognitive study. ${ }^{17}$ The absence of cognitive effects of mHT during and immediately after mHT is also consistent with the Early versus Late Intervention with Estradiol Study in recently menopausal women, which showed no cognitive effects of early menopausal $\mathrm{mHT}^{28}$ However, the absence of effects on global cognitive function by 7 years does not rule out potential long-term cognitive effects of the brain structural changes observed in the oCEE group during the mHT phase. Alteration of brain structure during midlife may influence cognitive outcomes later in life. ${ }^{29}$

Contrary to the changes in global brain volumes, the WMH volume showed a trend of increase both during and after the end of $\mathrm{mHT}$. This trend of increase in WMH volumes was present both in the oCEE and tE2 group. Although the magnitude of increase in WMH volume from baseline to month 84 was similar in the oCEE (mean increase $=0.08 \mathrm{~cm}^{3}$ ) and tE2 (mean increase $=0.07 \mathrm{~cm}^{3}$ ) groups, the rate of increase in $\mathrm{WMH}$ volume was statistically significantly greater than placebo only in the oCEE group. WMH are associated with hypertension and small vessel ischemic disease that may influence cognitive function in older adults. ${ }^{30-32}$ However, little is known about the significance of $\mathrm{WMH}$ volume in middle-aged women with good cardiovascular health. ${ }^{33}$ In the current KEEPS cohort, presence of a greater concentration of thrombogenic platelet-derived microvesicles in the blood at baseline was associated with a greater rate of increase in WMH volume during the mHT phase of the trial. ${ }^{22}$ It is possible that the thrombogenic properties of estrogens have influenced the rate of increase in WMH volume in women randomized to $\mathrm{mHT}$. The relationship of thrombogenic microvesicles to development of WMH after stopping mHT remains to be determined.

As expected in an aging cohort, the cortical volumes declined over the 7 years of follow-up. However, an unbiased regionbased analysis showed that the volume of the dorsolateral prefrontal cortex was preserved more in the tE2 group compared to placebo. Furthermore, greater preservation of the dorsolateral prefrontal cortical volume correlated with lower global cortical PiB uptake on PET only in the tE2 group. In an earlier study, we had observed lower global cortical PiB uptake in the $\mathrm{tE} 2$ group compared to placebo. ${ }^{19}$ In this study, we found an association between the lower PiB uptake (i.e., $\beta$-amyloid deposition) and the preservation of the dorsolateral prefrontal cortex volume in the $\mathrm{tE} 2$ group, suggesting that both may be influenced by the integrity of estrogen receptors $(\alpha$ and $\beta$ ) in the brain. Estradiol exerts its effects on the brain, such as maintaining synaptic integrity, ${ }^{34}$ and facilitating the production of soluble $\beta$-amyloid, through the estrogen receptors. ${ }^{35}$ For example, estrogen treatment increases the number of dendritic spine numbers in the prefrontal cortex and the hippocampus of ovariectomized female rhesus monkeys. ${ }^{36}$ With the reduction of estrogens after menopause, and with aging, the density of estrogen receptors decline. ${ }^{36}$ Administration of mHT soon after menopause may delay this decline in the estrogen receptors, and the effects of estrogens may be maintained during the early menopausal years. Furthermore, these effects may be agedependent, because the association of preservation of the dorsolateral prefrontal cortex and PiB uptake attenuated after adjusting for age in the current study.

Contrary to the observations in the tE2 group, regional changes in brain structure were not observed in the oCEE group. Furthermore, PiB uptake in the oCEE group was not different from placebo in the earlier study, ${ }^{19}$ suggesting that these effects of mHT on the brain does not extend to all formulations of mHT. It is expected that the various circulating products of estrogen metabolism in oCEE would have different efficacy in binding and activation of estrogen receptor-mediated events such as $\beta$-amyloid clearance and the production of soluble $\beta$-amyloid. ${ }^{35,37}$ For example, serum levels of estrone and of sulfonated conjugates are greater with oCEE than with tE2; therefore, the systemic effects and the receptor-mediated effects of oCEE on the brain may be different from the effects of tE2. Overall, the relative preservation of dorsolateral prefrontal cortical volume in the tE2 group over 7 years suggests that tE2 may have long-term effects on the brain.

In the WHIMS, women with type 2 diabetes, who received oCEE treatments, had a higher incidence of cognitive impairment $^{38}$ and greater loss of brain volume ${ }^{39}$ than nondiabetic women. Because KEEPS was conducted in recently menopausal women with good cardiovascular health, it was not possible to investigate the effects of type 2 diabetes or other cardiovascular risk factors on our findings. As such, the results may not be generalizable to women with high cardiovascular risk or type 2 diabetes. ${ }^{40}$ However, in this relatively healthy cohort of women, it was possible to directly observe the effects of mHT on the brain structure, without interference from contributing cardiovascular risk factors. Although we were able to retain $74 \%$ of the baseline KEEPSMRI study cohort over the 7 years of follow-up, this was a relatively small sample. Evaluation of a larger cohort of 
KEEPS participants to study the long-term effects of $\mathrm{mHT}$ on the brain and cognitive function is warranted.

\section{Author contributions}

Dr. Kantarci: design or conceptualization of the study, data collection, analysis and interpretation of the data, drafting the manuscript, study funding. Ms. Tosakulwong, Mr. Lesnick, Ms. Zuk, Ms. Settell, Dr. Gunter, Dr. Gleason, and Dr. Dowling: analysis or interpretation of the data, revising the manuscript. Dr. Fields: data collection, analysis or interpretation of the data, revising the manuscript. Mr. Senjem: analysis or interpretation of the data, revising the manuscript. Dr. Lowe and Dr. Shuster: data collection, analysis or interpretation of the data, revising the manuscript. Dr. Bailey, Dr. Rocca, Dr. Asthana, and Dr. Jack: analysis or interpretation of the data, revising the manuscript. Dr. Miller: design or conceptualization of the study, data collection, analysis or interpretation of the data, revising the manuscript, study funding.

\section{Study funding}

This study is funded by the Aurora Foundation to the Kronos Longevity Research Institute and the NIH (NS66147, AG029624, AG44170, and AG057547). The funding sources had no role in study design, collection, analysis, interpretation, or decision to submit this paper. The corresponding author had full access to all the data in the study and had final responsibility for the decision to submit for publication.

\section{Disclosure}

K. Kantarci serves on the data safety monitoring board for Takeda Global Research \& Development Center, Inc. She is funded by the NIH and receives research support from Avid/Eli Lilly Company. N. Tosakulwong, T. Lesnick, and S. Zuk report no disclosures relevant to the manuscript. V. Lowe consults for Bayer Schering Pharma, Piramal Life Sciences, and Merck Research and receives research support from GE Healthcare, Siemens Molecular Imaging, AVID Radiopharmaceuticals, and the NIH (NIA, NCI). J. Fields is funded by the NIH. J. Gunter reports no disclosures relevant to the manuscript. M. Senjem holds stock in Gilead Sciences, Inc., Inovio Pharmaceuticals, Medtronic, Oncothyreon, Inc., and PAREXEL International. M. Settell reports no disclosures relevant to the manuscript. C. Gleason is funded by the NIH. L. Shuster reports no disclosures relevant to the manuscript. K. Bailey is funded by the NIH. M. Dowling reports no disclosures relevant to the manuscript. $\mathrm{S}$. Asthana is funded by the NIH. C. Jack provides consulting services for Eli Lilly. He is funded by the NIH and Alexander Family Alzheimer's Disease professorship of the Mayo Foundation. W. Rocca is funded by the NIH. V. Miller is funded by NIH. Go to Neurology.org/N for full disclosures.

Received June 28, 2017. Accepted in final form January 18, 2018.

\section{References}

1. Espeland MA, Shumaker SA, Leng I, et al. Long-term effects on cognitive function of postmenopausal hormone therapy prescribed to women aged 50 to 55 years. JAMA Intern Med 2013;173:1429-1436.
2. LeBlanc ES, Janowsky J, Chan BK, Nelson HD. Hormone replacement therapy and cognition: systematic review and meta-analysis. JAMA 2001;285:1489-1499.

3. Prentice RL, Manson JE, Langer RD, et al. Benefits and risks of postmenopausal hormone therapy when it is initiated soon after menopause. Am J Epidemiol 2009;170:12-23.

4. Rocca WA, Grossardt BR, Shuster LT. Oophorectomy, estrogen, and dementia: a 2014 update. Mol Cell Endocrinol 2014;389:7-12.

5. Sherwin BB. Estrogen and memory in women: how can we reconcile the findings? Horm Behav 2005;47:371-375.

6. Waring SC, Rocca WA, Petersen RC, O’Brien PC, Tangalos EG, Kokmen E. Postmenopausal estrogen replacement therapy and risk of $\mathrm{AD}$ : a population-based study. Neurology 1999;52:965-970.

7. Whitmer RA, Quesenberry CP, Zhou J, Yaffe K. Timing of hormone therapy and dementia: the critical window theory revisited. Ann Neurol 2011;69:163-169.

8. Yaffe K, Sawaya G, Lieberburg I, Grady D. Estrogen therapy in postmenopausal women: effects on cognitive function and dementia. JAMA 1998;279:688-695.

9. Zandi PP, Carlson MC, Plassman BL, et al. Hormone replacement therapy and incidence of Alzheimer disease in older women: the Cache County Study. JAMA 2002;288:2123-2129; comment 2170-2172.

10. Shao H, Breitner JC, Whitmer RA, et al. Hormone therapy and Alzheimer disease dementia: new findings from the Cache County Study. Neurology 2012;79:1846-1852.

11. Bagger YZ, Tanko LB, Alexandersen P, Qin G, Christiansen C; PERF Study Group. Early postmenopausal hormone therapy may prevent cognitive impairment later in life. Menopause 2005;12:12-17.

12. Henderson VW, Benke KS, Green RC, Cupples LA, Farrer LA; MIRAGE Study Group. Postmenopausal hormone therapy and Alzheimer's disease risk: interaction with age. J Neurol Neurosurg Psychiatry 2005;76:103-105.

13. Imtiaz B, Tuppurainen M, Rikkonen T, et al. Postmenopausal hormone therapy and Alzheimer disease: a prospective cohort study. Neurology 2017;88:1062-1068.

14. Maki PM. A systematic review of clinical trials of hormone therapy on cognitive function: effects of age at initiation and progestin use. Ann NY Acad Sci 2005;1052. 182-197.

15. Harman SM, Brinton EA, Cedars M, et al. KEEPS: the Kronos Early Estrogen Prevention Study. Climacteric 2005;8:3-12.

16. Harman SM, Black DM, Naftolin F, et al. Arterial imaging outcomes and cardiovascular risk factors in recently menopausal women: a randomized trial. Ann Intern Med 2014;161:249-260.

17. Gleason CE, Dowling NM, Wharton W, et al. Effects of hormone therapy on cognition and mood in recently postmenopausal women: findings from the randomized, controlled KEEPS-Cognitive and Affective Study. PLoS Med 2015;12:e1001833; discussion e1001833.

18. Kantarci K, Tosakulwong N, Lesnick TG, et al. Effects of hormone therapy on brain structure: a randomized controlled trial. Neurology 2016;87:887-896.

19. Kantarci K, Lowe VJ, Lesnick TG, et al. Early postmenopausal transdermal $17 \beta$-estradiol therapy and amyloid- $\beta$ deposition. J Alzheimers Dis 2016;53:547-556.

20. Dowling NM, Gleason CE, Manson JE, et al. Characterization of vascular disease risk in postmenopausal women and its association with cognitive performance. PLoS One 2013;8:e68741.

21. Gunter JL, Shiung MM, Manduca A, Jack CR Jr. Methodological considerations for measuring rates of brain atrophy. J Magn Reson Imaging 2003;18:16-24.

22. Raz L, Jayachandran $\mathrm{M}$, Tosakulwong $\mathrm{N}$, et al. Thrombogenic microvesicles and white matter hyperintensities in postmenopausal women. Neurology 2013;80:911-918.

23. Cash DM, Frost C, Iheme LO, et al. Assessing atrophy measurement techniques in dementia: results from the MIRIAD atrophy challenge. Neuroimage 2015;123: 149-164.

24. Vemuri P, Whitwell JL, Kantarci K, et al. Antemortem MRI based STructural Abnormality iNDex (STAND)-scores correlate with postmortem Braak neurofibrillary tangle stage. Neuroimage 2008;42:559-567.

25. Jack CR Jr, Lowe VJ, Senjem ML, et al. $11 \mathrm{C} \mathrm{PiB} \mathrm{and} \mathrm{structural} \mathrm{MRI} \mathrm{provide}$ complementary information in imaging of Alzheimer's disease and amnestic mild cognitive impairment. Brain 2008;131:665-680.

26. Resnick SM, Espeland MA, Jaramillo SA, et al. Postmenopausal hormone therapy and regional brain volumes: the WHIMS-MRI Study. Neurology 2009;72:135-142.

27. Coker LH, Espeland MA, Hogan PE, et al. Change in brain and lesion volumes after CEE therapies: the WHIMS-MRI studies. Neurology 2014;82:427-434.

28. Henderson VW, St. John JA, Hodis HN, et al. Cognitive effects of estradiol after menopause: a randomized trial of the timing hypothesis. Neurology 2016;87: 699-708.

29. Vemuri P, Knopman DS, Lesnick TG, et al. Evaluation of amyloid protective factors and Alzheimer disease neurodegeneration protective factors in elderly individuals. JAMA Neurol 2017;74:718-726.

30. DeCarli C, Miller BL, Swan GE, Reed T, Wolf PA, Carmelli D. Cerebrovascular and brain morphologic correlates of mild cognitive impairment in the National Heart, Lung, and Blood Institute Twin Study. Arch Neurol 2001;58:643-647.

31. Silbert LC, Nelson C, Howieson DB, Moore MM, Kaye JA. Impact of white matter hyperintensity volume progression on rate of cognitive and motor decline. Neurology 2008;71:108-113.

32. Swan GE, DeCarli C, Miller BL, et al. Association of midlife blood pressure to late-life cognitive decline and brain morphology. Neurology 1998;51:986-993.

33. Barnes JN, Harvey RE, Zuk SM, et al. Aortic hemodynamics and white matter hyper intensities in normotensive postmenopausal women. J Neurol 2017;264:938-945.

34. Tang Y, Janssen WG, Hao J, et al. Estrogen replacement increases spinophilinimmunoreactive spine number in the prefrontal cortex of female rhesus monkeys. Cereb Cortex 2004;14:215-223. 
35. Jaffe AB, Toran-Allerand CD, Greengard P, Gandy SE. Estrogen regulates metabolism of Alzheimer amyloid beta precursor protein. J Biol Chem 1994;269:13065-13068.

36. Hara Y, Waters EM, McEwen BS, Morrison JH. Estrogen effects on cognitive and synaptic health over the lifecourse. Physiol Rev 2015;95:785-807.

37. Li R, Shen Y, Yang LB, Lue LF, Finch C, Rogers J. Estrogen enhances uptake of amyloid beta-protein by microglia derived from the human cortex. J Neurochem 2000;75:1447-1454.
38. Espeland MA, Brinton RD, Hugenschmidt C, et al. Impact of type 2 diabetes and postmenopausal hormone therapy on incidence of cognitive impairment in older women. Diabetes Care 2015;38:2316-2324.

39. Espeland MA, Brinton RD, Manson JE, et al. Postmenopausal hormone therapy, type 2 diabetes mellitus, and brain volumes. Neurology 2015;85:1131-1138.

40. Brinton RD. Neuroendocrinology: oestrogen therapy affects brain structure but not function. Nat Rev Neurol 2016;12:561-562. 


\section{Brain structure and cognition 3 years after the end of an early menopausal hormone therapy trial}

Kejal Kantarci, MD, MS, Nirubol Tosakulwong, Timothy G. Lesnick, MS, Samantha M. Zuk, Val J. Lowe, MD, Julie A. Fields, PhD, Jeffrey L. Gunter, PhD, Matthew L. Senjem, MS, Megan L. Settell, Carey E. Gleason, PhD, Lynne T. Shuster, MD, Kent R. Bailey, PhD, N. Maritza Dowling, PhD, Sanjay Asthana, MD, Clifford R. Jack, Jr., MD, Walter A. Rocca, MD, MPH, and Virginia M. Miller, PhD

Cite as: Neurology ${ }^{\circledR}$ 2018;90:e1404-e1412. doi:10.1212/WNL.0000000000005325

\section{Correspondence}

Dr. Kantarci

kantarci.kejal@mayo.edu

\section{Trial registration number}

NCT00154180 on ClinicalTrials.gov.

\section{Study question}

Does menopausal hormone therapy ( $\mathrm{mHT}$ ) have residual effects on brain structure and cognition 3 years after the end of a 4-year treatment period?

\section{Summary answer}

mHT exerts long-term effects on brain structure that persist for years after treatment cessation.

\section{Classification of evidence \\ Class III.}

\section{What is known and what this paper adds}

Estrogen-based mHT may preserve neurologic function and reduce the risk of dementia if applied early in menopause, but directly assessing this hypothesis would require decades of follow-up. This study analyzes noninvasive imaging biomarkers and provides evidence that $\mathrm{mHT}$ has persistent long-term effects on brain structure.

\section{Participants and setting}

This study analyzed 75 women (mean age 53 years) from the KEEPS study who underwent MRI assessments at the Mayo Clinic. The participants were free of neurologic disorders.

\section{Design, size, and duration}

This double-blind placebo-controlled trial randomized participants into groups receiving oral conjugated equine estrogens (oCEE; $0.45 \mathrm{mg} / \mathrm{d}$; $\mathrm{n}=20$ ), transdermal $17 \beta$-estradiol ( $\mathrm{tE} 2 ; 50 \mu \mathrm{g} / \mathrm{d} ; \mathrm{n}=22$ ), or placebo pills and patches $(n=33)$ for 4 years. The participants underwent MRI scans in months 18,36 , and 48 during the treatment period and underwent follow-up MRI scans 3 years after the treatment period (i.e., in month 84).

\section{Primary outcomes}

The primary outcomes were from-baseline changes in ventricular, whole-brain, and white matter hyperintensity (WMH) volumes at 84 months as revealed by MRI scans.

\section{Main results and the role of chance}

Relative to the placebo group, the oCEE group showed greater mean from-baseline increases in WMH volume at 84 months $(p=0.03)$, but the $\mathrm{tE} 2$ group did not $(p=0.17)$. The $\mathrm{mHT}$ groups did not differ from
Figure Longitudinal changes in white matter hyperintensity volumes

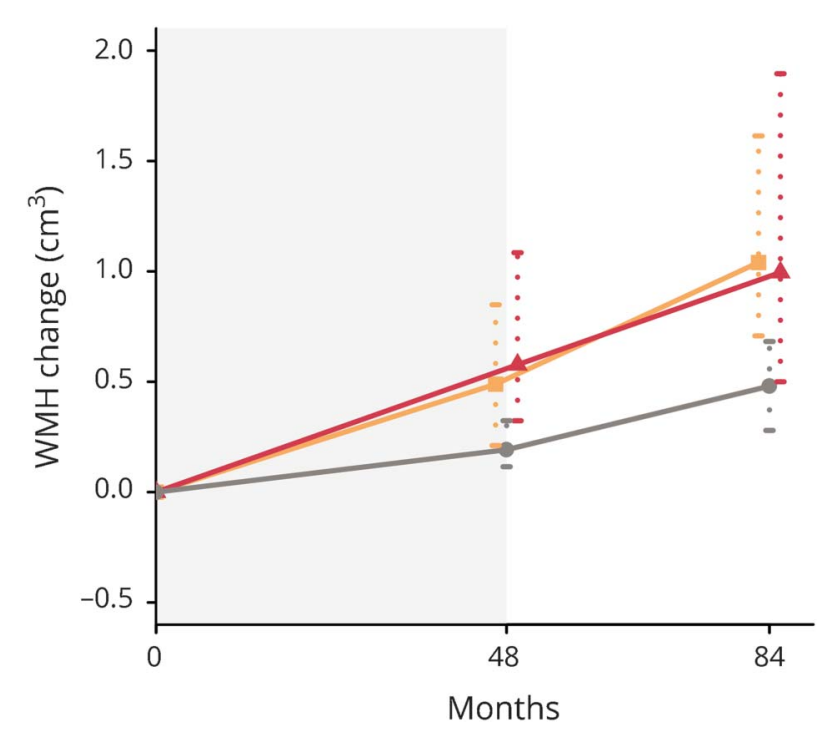

Gray circle $=$ placebo; orange square $=\mathrm{oCEE} ;$ red triangle $=\mathrm{tE} 2$.

the placebo group in from-baseline ventricular or whole-brain volume changes at 84 months.

\section{Harms}

Greater WMH volumes are associated with cerebrovascular conditions that may influence cognition in older adults.

\section{Bias, confounding, and other reasons for caution}

This study had a relatively small sample size.

\section{Generalizability to other populations}

The KEEPS participants were recently menopausal women in good cardiovascular health, so the results may not be generalizable to women in poor cardiovascular health or women with type 2 diabetes.

\section{Study funding/potential competing interests}

This study was funded by the Aurora Foundation and the NIH. Some authors report consulting for, holding stock in, receiving funding from, and serving on advisory boards for various healthcare companies. Go to Neurology.org/ $\mathrm{N}$ for full disclosures.

A draft of the short-form article was written by M. Dalefield, a writer with Editage, a division of Cactus Communications. The authors of the full-length article and the journal editors edited and approved the final version. 


\section{Neurology}

\section{Brain structure and cognition 3 years after the end of an early menopausal hormone therapy trial}

Kejal Kantarci, Nirubol Tosakulwong, Timothy G. Lesnick, et al.

Neurology 2018;90;e1404-e1412 Published Online before print March 21, 2018

DOI 10.1212/WNL.0000000000005325

\section{This information is current as of March 21, 2018}

Updated Information \&

Services

References

Subspecialty Collections

Permissions \& Licensing

Reprints including high resolution figures, can be found at: http://n.neurology.org/content/90/16/e1404.full

This article cites 40 articles, 14 of which you can access for free at: http://n.neurology.org/content/90/16/e1404.full\#ref-list-1

This article, along with others on similar topics, appears in the following collection(s):

Alzheimer's disease

http://n.neurology.org/cgi/collection/alzheimers_disease MRI

http://n.neurology.org/cgi/collection/mri

PET

http://n.neurology.org/cgi/collection/pet

Information about reproducing this article in parts (figures,tables) or in its entirety can be found online at:

http://www.neurology.org/about/about_the_journal\#permissions

Information about ordering reprints can be found online:

http://n.neurology.org/subscribers/advertise

Neurology ${ }^{\circledR}$ is the official journal of the American Academy of Neurology. Published continuously since 1951, it is now a weekly with 48 issues per year. Copyright Copyright ( 2018 The Author(s). Published by Wolters Kluwer Health, Inc. on behalf of the American Academy of Neurology. All rights reserved. Print ISSN: 0028-3878. Online ISSN: 1526-632X.

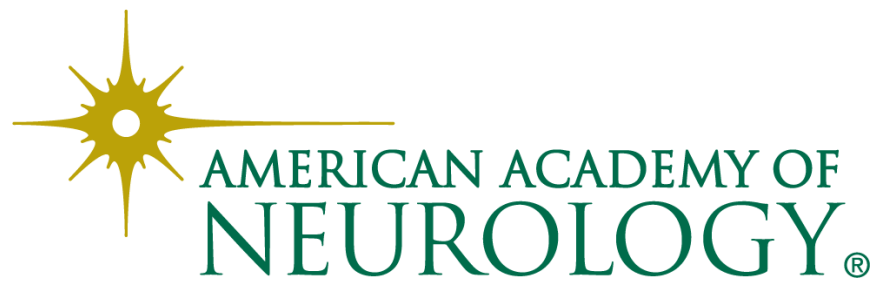

